\title{
Factors associated with readmission to the Emergency Department in a cohort of COVID-19 hospitalized patients
}

\author{
Álvaro Romero-Duarte ${ }^{1}$, Mario Rivera-Izquierdo ${ }^{2,3,4, *}$, \\ Antonio Jesús Láinez-Ramos-Bossini ${ }^{5,6}$, Pablo Redruello-Guerrero ${ }^{1}$, \\ Antonio Cárdenas-Cruz ${ }^{7,8}$
}

\author{
${ }^{1}$ Faculty of Medicine, University of \\ Granada, 18016 Granada, Spain \\ ${ }^{2}$ Service of Preventive Medicine and \\ Public Health, Hospital Universitario \\ Clínico San Cecilio, 18016 Granada, \\ Spain \\ ${ }^{3}$ Department of Preventive Medicine \\ and Public Health, University of \\ Granada, 18016 Granada, Spain \\ ${ }^{4}$ Instituto Biosanitario de Granada \\ (ibs.GRANADA), 37007 España, Spain \\ ${ }^{5}$ Department of Radiology, Virgen de las \\ Nieves University Hospital, 18016 \\ Granada, Spain \\ ${ }^{6} \mathrm{PhD}$ Programme in Clinical Medicine \\ and Public Health, University of \\ Granada, 18016 Granada, Spain \\ ${ }^{7}$ Department of Medicine, University of \\ Granada, 18016 Granada, Spain \\ ${ }^{8}$ Intensive Care Unit, Hospital de \\ Poniente, El Ejido, 04003 Almería, Spain
}

\section{*Correspondence}

mariorivera@ugr.es

(Mario Rivera-Izquierdo)

\begin{abstract}
Introduction: The aim of this study was to describe the symptomatology and main factors associated with readmission to the Emergency Department (ED) in COVID-19 patients discharged from hospital during the first wave of the pandemic at the San Cecilio University Hospital, Granada, Spain.

Methods: An observational longitudinal study was conducted in a cohort of 441 patients admitted to our hospital with confirmed SARS-CoV-2 polymerase chain reaction (PCR) from 1 March to 15 April 2020. Patients were followed up through medical records 6 months after discharge. Sociodemographic, clinical and symptomatologic variables were collected. Descriptive, bivariate and multivariate logistic regression analyses were performed.

Results: The mean age of patients in the cohort was 66.4 years ( $\mathrm{s}=15.3$ ), with $55.1 \%$ men. In-hospital mortality was $18.1 \%$. The presence of persistent symptomatology was high (64.5\%), especially respiratory (53.2\%), systemic (46.3\%) and neurological $(31.0 \%)$. A total of 75 (20.8\%) patients were readmitted to the ED during the 6 months following hospital discharge. The main factors associated with readmission to the ED were polymedication $(P=0.031)$, living in a care home $(P=0.014)$, fever $(P=$ 0.047), general malaise $(P<0.001)$, thoracic pain $(P<0.001)$, headache $(P=0.012)$, hematological symptoms $(P=0.011)$, nephrological symptoms $(P=0.047)$, depressive symptoms $(P=0.009)$, syncope or hypotension $(P=0.006)$ and superinfection $(P=$ 0.018 ). After multivariate adjustment analysis, thoracic pain (OR: $4.45,95 \%$ CI: 1.88 10.52), general malaise and hematological symptoms (OR: 3.95, 95\% CI: 1.12-13.89) remained as risk factors.

Conclusions: The presence of persistent symptomatology after hospital discharge in our cohort was common and varied. Polymedication and living in a care home made up the most vulnerable profile of COVID-19 patients for returning to the ED. Thoracic pain, general malaise and hematological symptoms were identified as potential markers of severity, along with others predictors. These findings might be useful for optimizing follow-up strategies. Future studies conducted in other geographical areas are necessary to corroborate our results.
\end{abstract}

\section{Keywords}

COVID-19; Symptoms; Emergency; Hospitalization; Post-discharge

\section{Introduction}

Since the identification of the first cases of COVID-19 [1-3], the rapid spread of the virus led to great efforts to identify the main prognostic factors of the disease and optimize preventive and therapeutic strategies during the pandemic. Rapid humanto-human transmission [2] along with high initial mortality and hospitalization rates [4] generated great concern and led to the development of unprecedented sanitary and economic measures worldwide. Older age and previous severe diseases have been reported to be the main prognostic factors, making up the most vulnerable population profile which is especially frequent in care homes [4]. Hypertension, diabetes mellitus, stroke or chronic lung disease represented the most frequent comorbidities of severe cases during the first wave of the pandemic [5].

The identification of factors associated with negative outcomes (e.g., in-hospital mortality, admission to Intensive Care 
Unit (ICU), sequelae, readmission to emergency care services, hospitalization or post-discharge death) is emerging as a specific field of research aim at individualizing follow-up strategies in these patients [6]. However, to date there is a lack of scientific literature on patient outcomes after discharge. Preventive strategies focused on reducing these outcomes should be based on follow-up studies. Apart from the most commonly known prognostic factors (age and comorbidities), recognizing less frequent risk factors is still needed [7], especially if they are modifiable and, consequently, preventable. Treatmentrelated factors are also becoming a major field of ongoing research to improve patient management [8].

Emergency Departments in Spain have played an important role in the early identification of severe COVID-19 cases, although in times of great healthcare pressure they have been overloaded [4, 8]. Identification of factors that may predict readmission to emergency care settings could help improve the follow-up of these patients in Primary Care services and the criteria for hospital discharge. For instance, sequelae or persistent symptomatology, also known as post-discharge syndrome [9], could help identify these recurrent cases. Several studies point to the presence of specific symptoms in the short to medium term, including neurological [10-12], cardiovascular [13, 14] or thrombotic [15] sequelae, which were described in the first follow-up studies. Nonetheless, the number of studies with longer follow-up periods is very low. In fact, to the best of our knowledge, only one study conducted in China has completed a 6-month follow-up to date [16]. In Spain, no studies analyzing long-term symptomatology following hospital discharge in COVID-19 patients have been conducted so far.

The aim of this study was twofold: to describe the symptomatology of COVID-19 patients after hospital discharge in our institution; and to analyze the factors (including symptoms) associated with readmission to the ED during the 6 months following discharge.

\section{Materials and methods}

\subsection{Design and setting}

This is an observational longitudinal retrospective study, conducted in a cohort of 441 patients who required hospitalization due to COVID-19 in the San Cecilio University Hospital (Granada, Spain) during the first wave of the pandemic, from 1 March to 15 April 2020. According to the inclusion criteria, only patients with confirmed positive polymerase chain reaction (PCR) to SARS-CoV-2 were considered in the study. The sample was followed up for 6 months from the date of discharge, thus follow-up finished on 10 January 2021.

\subsection{Data source and variables}

Information was collected from medical records of hospitalization and follow-up reports from Primary Care institutions during the 6 months following hospital discharge. All data were gathered by the same researcher (AR-D) to avoid heterogeneity of information and reviewed by two experienced researchers (MR-I and AC-C). The variables considered for the analyses were:

- Sociodemographic variables: sex, age, residence, de- pendence in activities of daily living.

- Clinical variables: comorbidities, laboratory workup, prognostic scores (CURB-65), treatment received, in-hospital mortality.

- Post-discharge symptomatology: information on symptoms during the 6 months after discharge were collected by means of periodic telephone consultations in Primary Care centers. These symptoms were classified into the following categories:

- Systemic symptoms: fever, asthenia, muscle weakness, musculoskeletal pain, general malaise.

- Respiratory symptoms: dyspnea, rib pain, thoracic pain, persistent cough, pharyngeal symptoms.

- Neurological symptoms: polyneuropathy, headache, sensitivity disorders, movement disorders, confusion, persistent anosmia or dysgeusia.

- Mental health symptoms: depressive, anxiety or sleep disorders.

- Hematological symptoms: anemia, thrombotic disorders.

- Dermatological symptoms: eczema, exanthema, pruritus, alopecia.

- Nephrological symptoms: renal failure.

- Urological symptoms: voiding syndrome.

- Ophthalmologic symptoms.

- Digestive symptoms: nausea, vomiting, diarrhea, constipation, anorexia, abdominal pain.

- Syncope or hypotension.

- Infectious symptoms: superinfections.

- Outcome: readmission to the ED within 6 months after hospital discharge.

\subsection{Statistical analyses}

First, a univariate descriptive analysis was performed. Then, bivariate analyses were applied using Pearson's chi-squared and Student's $T$-tests if the assumptions of parametric testing were met (otherwise, non-parametric tests were applied, e.g., Fisher's exact test). The dependent variable of our study was the primary outcome, i.e., readmission to the ED during 6 months after discharge. Finally, multivariate analyses for predicting the outcome were performed using logistic regression models. The estimates of strength of association were calculated as odds ratios (ORs). All analyses were performed using the SPSS ${ }^{\circledR}$ version 22.0 statistical software.

\subsection{Ethical considerations}

This study complies with the principles of the Declaration of Helsinki. A completely anonymized database was used for the analyses. No identification data were used. The study was approved by the Provincial Research Ethics Committee of Granada on 1 October 2020 (code 1585-N-20).

\section{Results}

\subsection{Descriptive analysis}

Of the total sample of hospitalized patients $(n=441$; mean age, 66.4 years; $55.1 \%$ men), $361(81.9 \%)$ survived and were 
TA B L E 1. Sociodemographic and clinical variables collected at baseline (hospital admission) in the followed sample.

\begin{tabular}{|c|c|c|c|c|}
\hline Sociodemographic variables & $\begin{array}{l}\text { Total followed-up cohort } \\
\qquad(\mathrm{n}=361)\end{array}$ & $\begin{array}{l}\text { Readmission to the ED } \\
\qquad(\mathrm{n}=75)\end{array}$ & $\begin{array}{l}\text { No readmission to the ED } \\
\qquad(\mathrm{n}=286)\end{array}$ & $P$-value ${ }^{a}$ \\
\hline & $\mathrm{N}(\%) / \mathrm{x}(\mathrm{s})$ & $\mathrm{N}(\%) / \mathrm{x}(\mathrm{s})$ & $\mathrm{N}(\%) / \mathrm{x}(\mathrm{s})$ & \\
\hline Age & $63.4(14.7)$ & $62.4(15.5)$ & $63.7(14.4)$ & 0.495 \\
\hline Men & $191(52.9)$ & $39(52.0)$ & $152(53.1)$ & 0.859 \\
\hline Hypertension & $163(45.2)$ & $28(37.3)$ & $135(47.2)$ & 0.126 \\
\hline Diabetes mellitus & $70(19.4)$ & $16(21.3)$ & $54(18.9)$ & 0.633 \\
\hline Chronic kidney disease & $28(7.8)$ & $7(9.3)$ & $21(7.3)$ & 0.566 \\
\hline Cardiovascular disease & $59(16.3)$ & $14(18.7)$ & $45(15.7)$ & 0.541 \\
\hline COPD & $17(4.7)$ & $2(2.7)$ & $15(5.2)$ & 0.348 \\
\hline Asthma & $30(8.3)$ & $9(12.0)$ & $21(7.3)$ & 0.193 \\
\hline Autoimmune disease & $22(6.1)$ & $8(10.7)$ & $14(4.9)$ & 0.063 \\
\hline Active neoplasm & $6(1.7)$ & $2(2.7)$ & $4(1.4)$ & 0.608 \\
\hline Polymedication & $130(36.0)$ & $35(46.7)$ & $95(33.2)$ & $0.031 *$ \\
\hline Dependence for ADL & $65(18.0)$ & $19(25.3)$ & $46(16.1)$ & 0.064 \\
\hline Living at home & $307(85.0)$ & $57(76.0)$ & $250(87.4)$ & $0.014 *$ \\
\hline Living in $\mathrm{RCH}$ & $39(10.8)$ & $12(16.0)$ & $27(9.4)$ & 0.103 \\
\hline Living in RCD & $18(5.0)$ & $5(6.7)$ & $13(4.5)$ & 0.453 \\
\hline \multicolumn{5}{|l|}{ Clinical variables } \\
\hline CRS & $127(35.2)$ & $24(32.9)$ & $103(36.1)$ & 0.603 \\
\hline Concomitant infection & $70(19.4)$ & $19(25.7)$ & $51(18.3)$ & 0.160 \\
\hline \multicolumn{5}{|l|}{ CURB-65 score } \\
\hline 0 & $106(29.4)$ & $22(30.6)$ & $84(30.2)$ & \multirow{4}{*}{0.845} \\
\hline 1 & $150(41.6)$ & $28(38.9)$ & $122(43.9)$ & \\
\hline 2 & $82(22.7)$ & $19(26.4)$ & $63(22.7)$ & \\
\hline 3 & $12(3.3)$ & $3(4.2)$ & $9(3.2)$ & \\
\hline Not candidate for CPR & $71(19.7)$ & $18(25.0)$ & $53(19.0)$ & 0.258 \\
\hline Hospitalization days & $16.1(13.3)$ & $18.6(14.7)$ & $15.4(12.9)$ & 0.062 \\
\hline ICU admission & $32(8.9)$ & $9(12.0)$ & $23(8.0)$ & 0.283 \\
\hline
\end{tabular}

Data are presented as absolute frequencies $(N)$ and relative frequencies (\%) for qualitative variables, and as mean ( $x$ ) and standard deviation (s) for quantitative variables. ED, Emergency Department; ADL, activities of daily living; COPD, chronic obstructive pulmonary disease; $R C H$, residential care homes for the elderly; RCD, residential care homes for adults with disability; CRS, cytokine release syndrome; CPR, cardiopulmonary resuscitation; ICU, intensive care unit. ${ }^{a} P$-values are calculated as T-test for quantitative variables and chi-square test for qualitative variables. When conditions of application were not met, Fisher exact test was used. *P<0.05.

discharged. This group comprised the sample followed (mean age, 62.4 years; $52.9 \%$ men). A total of $75(20.8 \%)$ patients were readmitted to the ED within 6 months after hospital discharge. The main characteristics of the subgroups of the cohort are described in Fig. 1. Table 1 shows the distribution of the sociodemographic and clinical variables of the followed sample, where the 'total followed-up cohort' column presents all the patients who were discharged alive, 'readmission to the ED' explores the subgroup of patients who were readmitted to the ED within the 6-month follow-up after discharge, and 'no readmission to the ED' describes the subgroup of patients who did not return to the ED during the said follow-up.

\subsection{Bivariate analyses}

As shown in Table 1, no significant differences in most of the sociodemographic or clinical variables at baseline were associated with readmission to the ED at 6 months, although a higher presence of comorbidities in the cohort of patients readmitted to the ED is observed for most of the analyzed variables. Conversely, not living at home and polymedication were significantly associated with the primary outcome. Table 2 shows the presence of symptoms during the 6-month follow-up after discharge of survivors $(n=361)$, stratified by the primary outcome. The 'readmission to the ED' column explores the subgroup of patients who returned to the ED during followup, and 'no readmission to the ED' describes the subgroup of 




F I G U R E 1. Main characteristics (percentage, sex and age) of the subgroups of the cohort.

patients who were not readmitted to the ED during follow-up.

As shown in the table, the most frequent symptoms were respiratory, systemic, neurological, and digestive.

Bivariate analyses showed that the factors potentially associated with readmission to the ED were fever, general malaise, thoracic pain, headache, hematological and nephrological symptoms, syncope or hypotension and superinfection. On the other hand, anosmia or dysgeusia and asthenia were associated with a lower frequency of readmission to the ED at 6 months.

\subsection{Multivariate analysis}

Multivariate logistic regression models were applied for predicting readmission to the ED (dependent variable). Models were adjusted for sex, age and the variables resulting from the bivariate analyses (Table 3 ). The 'cOR' column shows the crude associations between each variable and the odds of returning to the ED during follow-up, and the 'aOR' column shows the adjusted associations in the multivariate models.

After adjustment of the models, the variables that remained as risk factors for readmission to the ED at 6 months following hospital discharge were polymedication, not living at home, general malaise and thoracic pain. Fig. 2 shows the distribution of the main factors associated with readmission to the ED in the multivariate analysis.

\section{Discussion}

In this study we described the frequency of symptoms during 6 months after discharge of COVID-19 patients in a tertiary hospital from Granada, Spain. We detailed the observed symptomatology and variables associated with readmission to the ED. Statistically significant associations were found between polymedication and living in residential care homes, and a higher frequency of readmission to the ED, which remarks the necessity of strengthening the follow-up of this group of vulnerable patients after hospital discharge during the pandemic.

Regarding the symptomatology analyzed in the cohort, general malaise and thoracic pain were associated with a higher frequency of readmission to the ED. In addition, bivariate analyses showed that headache, syncope or hypotension and superinfection were also associated with higher ED readmission rates. Accordingly, we suggest that these symptoms should serve as guidance for physicians in the follow-up of postdischarge COVID-19 patients to avoid hospital readmission or negative outcomes.

The mean age (63.4 years) and sex distribution (52.9\% of men) of our sample are very similar to other previous studies conducted in our environment $[4,8]$. The in-hospital mortality of our cohort $(18.1 \%)$ is also in agreement with data from large cohorts in other countries during the first wave of the pandemic, such as the United States [17]. However, it is slightly lower compared to data from studies published in Wuhan [18]. This difference might be partially explained by 
T A B L E 2. Symptomatology during the 6 months after hospital discharge of COVID-19 patients.

\begin{tabular}{|c|c|c|c|c|}
\hline Symptoms & $\begin{array}{l}\text { Total followed cohort } \\
\qquad(\mathrm{n}=361)\end{array}$ & $\begin{array}{l}\text { Readmission to the ED } \\
\qquad(\mathrm{n}=75)\end{array}$ & $\begin{array}{l}\text { No readmission to the ED } \\
\qquad(\mathrm{n}=286)\end{array}$ & $P$-value ${ }^{a}$ \\
\hline & $\mathrm{N}(\%) / \mathrm{x}(\mathrm{s})$ & $\mathrm{N}(\%) / \mathrm{x}(\mathrm{s})$ & $\mathrm{N}(\%) / \mathrm{x}(\mathrm{s})$ & \\
\hline Any symptom & $233(64.5)$ & $52(69.3)$ & $182(63.6)$ & 0.670 \\
\hline Systemic symptoms & $167(46.3)$ & $40(53.3)$ & $127(44.4)$ & 0.168 \\
\hline Fever & $32(8.9)$ & $11(14.7)$ & $21(7.3)$ & $0.047^{*}$ \\
\hline Asthenia & $110(30.5)$ & $16(21.2)$ & $94(32.9)$ & 0.053 \\
\hline Muscle weakness & $27(7.5)$ & $8(10.7)$ & $19(6.6)$ & 0.238 \\
\hline Musculoskeletal pain & $69(19.1)$ & $19(25.3)$ & $50(17.5)$ & 0.124 \\
\hline General malaise & $18(5.0)$ & $10(13.3)$ & $8(2.8)$ & $<0.001 *$ \\
\hline Respiratory symptoms & $192(53.2)$ & $45(60.0)$ & $147(51.4)$ & 0.184 \\
\hline Dyspnea & $137(38.0)$ & $32(42.7)$ & $105(36.7)$ & 0.344 \\
\hline Rib pain & $19(5.3)$ & $7(9.3)$ & $12(4.2)$ & 0.076 \\
\hline Thoracic pain & $30(8.3)$ & $15(20.0)$ & $15(5.2)$ & $<0.001^{*}$ \\
\hline Persistent cough & $83(23.0)$ & $15(20.0)$ & $68(23.8)$ & 0.489 \\
\hline Pharyngeal symptoms & $39(10.8)$ & $10(13.3)$ & $29(10.1)$ & 0.428 \\
\hline Neurological symptoms & $112(31.0)$ & $26(34.7)$ & $86(30.1)$ & 0.444 \\
\hline Headache & $28(7.8)$ & $11(14.7)$ & $17(5.9)$ & $0.012 *$ \\
\hline Sensitivity disorders & $19(5.3)$ & $7(9.3)$ & $12(4.2)$ & 0.076 \\
\hline Movement disorders & $18(5.0)$ & $5(6.7)$ & $13(4.5)$ & 0.453 \\
\hline Confusion & $13(3.6)$ & $5(6.7)$ & $8(2.8)$ & 0.109 \\
\hline Anosmia or dysgeusia & $46(12.7)$ & $4(5.3)$ & $42(14.7)$ & $0.031 *$ \\
\hline Mental health symptoms & $67(18.6)$ & $18(24.0)$ & $49(17.1)$ & 0.173 \\
\hline Depressive symptoms & $24(6.6)$ & $10(13.3)$ & $14(4.9)$ & $0.009 *$ \\
\hline Anxiety & $33(9.1)$ & $10(13.3)$ & $23(8.0)$ & 0.157 \\
\hline Sleep disorders & $32(8.9)$ & $9(12.0)$ & $23(8.9)$ & 0.283 \\
\hline Hematological symptoms & $12(3.3)$ & $6(8.0)$ & $6(2.1)$ & $0.011 *$ \\
\hline Thrombotic signs & $9(2.5)$ & $4(5.3)$ & $5(1.7)$ & 0.076 \\
\hline Dermatological symptoms & $38(10.5)$ & $11(14.7)$ & $27(9.4)$ & 0.189 \\
\hline Exanthema & $10(2.8)$ & $4(5.3)$ & $6(2.1)$ & 0.129 \\
\hline Nephrological symptoms & $32(8.9)$ & $11(14.7)$ & $21(7.3)$ & $0.047^{*}$ \\
\hline Urological symptoms & $15(4.2)$ & $2(2.7)$ & $13(4.5)$ & 0.468 \\
\hline Ophthalmologic symptoms & $21(5.8)$ & $7(9.3)$ & $14(4.9)$ & 0.144 \\
\hline Digestive symptoms & $81(22.4)$ & $22(29.3)$ & $59(20.6)$ & 0.108 \\
\hline Diarrhea & $54(15.0)$ & $11(14.7)$ & $43(15.0)$ & 0.937 \\
\hline Abdominal pain & $21(5.8)$ & $7(9.3)$ & $14(4.9)$ & 0.144 \\
\hline Syncope or hypotension & $17(4.7)$ & $8(10.7)$ & $9(3.1)$ & $0.006^{*}$ \\
\hline Superinfection & $29(8.0)$ & $11(14.7)$ & $18(6.3)$ & $0.018 *$ \\
\hline Post-discharge mortality & $4(1.1)$ & $2(2.7)$ & $2(0.7)$ & 0.147 \\
\hline
\end{tabular}

Data are presented as absolute frequencies ( $N$ ) and relative frequencies (\%) for qualitative variables, and as mean ( $x$ ) and standard deviation (s) for quantitative variables. ${ }^{a} P$-values are calculated as T-tests for quantitative variables and chi-square tests for qualitative variables. When conditions of application were not met, Fisher's exact test was applied. ${ }^{*} P<0.05$.

the design of the studies. We conducted a complete followup of a cohort of patients who had been hospitalized, while studies on mortality published during the first months of the pandemic such as [18] only considered the primary outcomes (death or discharge) with no complete follow-up. Accordingly, these studies likely underestimated cases of long-hospital stay and overestimated the mortality rates. Other studies [19] have attempted to characterize COVID-19 patients who returned to 
च \% of patients readmitted to the Emergency Department $\quad \square \%$ of patients who were not readmitted to the Emergency Department

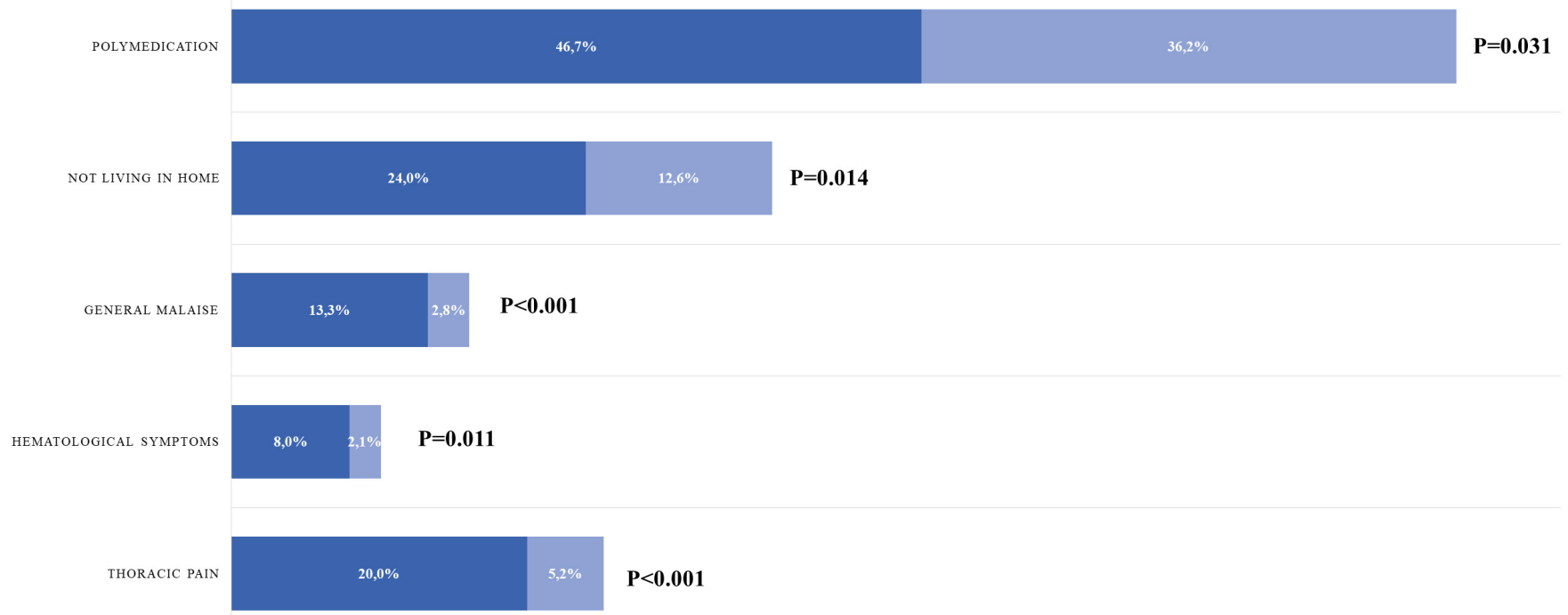

FIGURE 2. Main factors associated with readmission to the Emergency Department in the multivariate analysis. Percentage distribution and $P$-values.

TA B L E 3. Multivariate logistic regression analysis for the outcome 'readmission to the Emergency Department during 6 months after hospital discharge'.

\begin{tabular}{lcc} 
Variable & COR $(95 \% \mathrm{CI})$ & $\mathrm{aOR}(95 \% \mathrm{CI})$ \\
Age & $0.99(0.98-1.01)$ & $0.98(0.95-1.00)$ \\
Sex (reference: men) & $1.05(0.63-1.74)$ & $0.85(0.48-1.51)$ \\
Polymedication & $1.75(1.11-2.76)$ & $2.12(1.16-3.89)^{*}$ \\
Not living at home & $2.19(1.16-4.14)$ & $2.51(1.16-5.48)^{*}$ \\
Fever & $2.17(1.00-4.73)$ & $1.37(0.56-3.32)$ \\
General malaise & $5.34(2.03-14.08)$ & $5.24(1.72-15.94)^{*}$ \\
Thoracic pain & $4.52(2.09-9.74)$ & $4.45(1.86-10.63)^{*}$ \\
Headache & $2.72(1.21-6.09)$ & $1.79(0.68-4.68)$ \\
Depressive symptoms & $2.99(1.27-7.03)$ & $2.38(0.88-6.47)$ \\
Hematological symptoms & $4.06(1.27-12.97)$ & $3.95(1.12-13.89)^{*}$ \\
Nephrological symptoms & $2.17(1.00-4.73)$ & $1.33(0.446-3.85)$ \\
Syncope or hypotension & $3.67(1.37-9.88)$ & $1.44(0.42-4.89)$ \\
Superinfection & $2.56(1.15-5.68)$ & $1.98(0.71-5.51)$ \\
\hline cOR, crude odds ratio (unadjusted); aOR, odds ratio adjusted for all the variables shown \\
in the table.
\end{tabular}

hospital following discharge. These authors found a $3.6 \%$ rate of return to the ED and agreed that the most frequent causes were comorbidities (especially hypertension and respiratory conditions) and respiratory distress. Conversely, we did not find associations with respiratory symptoms but with thoracic pain. Other authors found that $8.6 \%$ of patients were readmitted to hospital or ED in the 72 hours after discharge [20]. The main factors associated with readmission were older age, hypoxia, fever, obesity and hypertension. We observed no association between respiratory symptoms and readmission, which contrasts with the results of studies conducted in the United States [19, 20]. This could be partially explained by the subjective report of symptoms in our study (self-reported rather than measurement of hypoxia, as was the case in previous studies). However, we agree that patient vulnerability (older age, comorbidities, dependence or polymedication) increases the frequency of post-discharge readmission to the ED and might be a potential risk factor for improving preventive follow-up strategies.

\subsection{Symptomatology}

The frequency of persistent symptoms during 6 months after hospital discharge in our study was surprisingly high. Almost 
two thirds of the patients $(64.5 \%)$ showed at least one symptom at follow-up. The most frequent symptoms identified in this study were respiratory (63.0\%), especially dyspnea (44.9\%); systemic $(54.9 \%)$, especially asthenia $(36.1 \%)$; neurological (36.7\%); digestive (26.6\%), especially diarrhea (17.7\%); and related to mental health $(22.0 \%)$.

The high frequency of potentially severe symptoms such as superinfection $(8.0 \%)$, hypotension $(4.7 \%)$ and hematological (3.3\%) symptoms should also be considered.

In addition, the high frequency (10.5\%) of dermatological symptoms associated with COVID-19 is in agreement with other studies [21, 22].

\subsection{Factors associated with readmission to the Emergency Department}

Readmission to the ED is an important concern which, considering the state-of-the-art literature on COVID-19, merits further research due to a number of reasons. First, during the pandemic, EDs have been overloaded due to an excessive healthcare demand. Second, readmission to the ED during the 6 months after hospital discharge might be a marker of severity in the short-to-medium term and possibly requires an optimization of the criteria used for hospital discharge or follow-up protocols of these patients by Primary Care physicians. We believe that the identification of factors associated with this severity might be useful for individualizing medical advice and information to the community.

According to the results of bivariate analyses, the main factors associated with readmission to the ED in our study were polymedication $(P=0.031)$, living in a residential care home $(P=0.014)$, fever $(P=0.047)$, general malaise $(P<0.001)$, thoracic pain $(P<0.001)$, headache $(P=0.012)$, depressive symptoms $(P=0.009)$, hematological symptoms $(P=0.011)$, nephrological symptoms $(P=0.047)$, syncope or hypotension $(P=0.006)$ and superinfection $(P=0.018)$. On the other hand, our results point to persistent anosmia or dysgeusia as a possible protective factor for readmission to the $\operatorname{ED}(P=$ $0.031)$. It is possible that these symptoms are more frequent in less severe cases of COVID-19. However, studies specifically aimed at contrasting this hypothesis should be conducted.

We believe that polymedication (more than six drugs per day) and living in residential care homes point to a vulnerable profile of patients that might require strengthening and individualizing post-discharge follow-up strategies. Both factors were confirmed by multivariate analyses (OR: $2.12,95 \% \mathrm{CI}$ : 1.16-3.89 and OR: 2.51, 95\% CI: 1.16-5.48, respectively).

Regarding symptoms, multivariate analyses confirmed the associations between higher frequency of readmission to the ED and general malaise (OR: 5.24, 95\% CI: 1.72-15.94), thoracic pain (OR: $4.45,95 \%$ CI: $1.86-10.63$ ) and hematological symptoms (OR: 3.95, 95\% CI: 1.12-13.89). However, given the limitations our study data, headache, depressive symptoms, nephrological symptoms, syncope or hypotension and superinfection should also be cautiously considered as possible markers of severity.

\subsection{Limitations of the study}

The primary aim of this study was to offer a global description of symptomatology during 6 months after discharge of COVID-19 in our institution. However, we could not gather information on the time of symptom onset, thus it was not possible to distinguish between early or late symptoms, and survival analyses or Cox regression models could not be performed. We used medical records for data gathering. Nevertheless, many potentially relevant variables such as obesity or smoking were not systematically collected in the reports and, therefore, could not be analyzed. We tried to minimize this limitation by collecting all available sociodemographic variables and including them in the multivariate analysis adjustments. It should also be noted that data were obtained from a single institution, thus the external validity of our results should be interpreted cautiously. Finally, possible biases associated with the retrospective nature of this study should be considered. We believe that the detailed information on symptoms and factors associated with readmission to the ED after 6 months of followup may contribute significantly to improving future follow-up of COVID-19 patients discharged from hospital.

\section{Conclusions}

The frequency of symptoms after hospital discharge in COVID-19 patients is high and variable. The most common symptoms were respiratory, systemic, neurological, digestive and related to mental health. Variables associated with readmission to the ED included polymedication, living in residential care homes, general malaise, thoracic pain and hematologic symptoms. These factors, along with headache, depressive symptoms, nephrological symptoms, syncope or hypotension, and superinfection might be used as markers of severity for improving follow-up of patients after hospital discharge. However, considering the limitations of our study, these results should be confirmed by long-term longitudinal studies conducted in other countries.

\section{AUTHOR CONTRIBUTIONS}

ARD and MRI designed the research study and wrote the first draft of the manuscript. ARD, MRI, AJLRB, PRG and ACC conducted the research and advised on methodology. ACC mentored the work. All authors contributed to editorial changes in the manuscript. All authors read and approved the final manuscript.

\section{ETHICS APPROVAL AND CONSENT TO PARTICIPATE}

This study complies with the principles of the Declaration of Helsinki. A completely anonymized database was used for the analyses. No identification data were used. The study was approved by the Provincial Research Ethics Committee of Granada on 1 October 2020 (code 1585-N-20). 


\section{ACKNOWLEDGMENT}

This work was supported by the 'Artificial Intelligence for the diagnosis and prognosis of COVID-19' project (CV20-29480), funded by the Consejería de Transformación Económica, Industria, Conocimiento y Universidades, Junta de Andalucía, and the FEDER funds. We acknowledge the Faculty of Medicine, University of Granada, for the successful organization of the final degree projects, since this work was designed and conducted during the project of Álvaro Romero-Duarte. We also acknowledge all the healthcare workers from the Department of Preventive Medicine and Public Health, San Cecilio University Hospital for their restless commitment during the pandemic of COVID-19 and their continuous efforts for investigating and communicating their results to the scientific community. Finally, we thank the SEMERGEN-UGR Chair of Teaching and Research in Family Medicine for being an example of support and encouragement in Primary Care research.

\section{FUNDING}

This study received no external funding.

\section{CONFLICT OF INTEREST}

The authors declare no conflict of interest. Antonio Jesús Láinez-Ramos-Bossini is a Guest Editor of this journal.

\section{REFERENCES}

[1] Lu R, Zhao X, Li J, Niu P, Yang B, Wu H, et al. Genomic characterisation and epidemiology of 2019 novel coronavirus: implications for virus origins and receptor binding. Lancet. 2020; 395: 565-574.

[2] Phan LT, Nguyen TV, Luong QC, Nguyen TV, Nguyen HT, Le HQ, et al. Importation and Human-to-Human Transmission of a Novel Coronavirus in Vietnam. New England Journal of Medicine. 2020; 382: 872-874.

[3] Holshue ML, DeBolt C, Lindquist S, Lofy KH, Wiesman J, Bruce H, et al. First Case of 2019 Novel Coronavirus in the United States. New England Journal of Medicine. 2020; 382: 929-936.

[4] Rivera-Izquierdo M, Valero-Ubierna MC, R-delAmo JL, FernándezGarcía MA, Martínez-Diz S, Tahery-Mahmoud A, et al. Sociodemographic, clinical and laboratory factors on admission associated with COVID-19 mortality in hospitalized patients: A retrospective observational study. PLoS ONE. 2020; 15: e0235107.

[5] Guan W, Ni Z, Hu Y, Liang W, Ou C, He J, et al. Clinical Characteristics of Coronavirus Disease 2019 in China. New England Journal of Medicine. 2020; 382: 1708-1720.

[6] Zheng Z, Peng F, Xu B, Zhao J, Liu H, Peng J, et al. Risk factors of critical \& mortal COVID-19 cases: a systematic literature review and meta-analysis. Journal of Infection. 2020; 81: e16-e25.

[7] Izcovich A, Ragusa MA, Tortosa F, Lavena Marcio MA, Agnoletti C, Bengolea A, et al. Prognostic factors for severity and mortality in patients infected with COVID-19: A systematic review. PLoS ONE. 2020; 15: $\mathrm{e} 0241955$.
${ }^{[8]}$ Rivera-Izquierdo M, Valero-Ubierna MDC, R-delAmo JL, FernándezGarcía MA, Martínez-Diz S, Tahery-Mahmoud A, et al. Therapeutic agents tested in 238 COVID-19 hospitalized patients and their relationship with mortality Medicina ClíNica. 2020; 155: 375-381.

[9] Garrigues E, Janvier P, Kherabi Y, Le Bot A, Hamon A, Gouze H, et al. Post-discharge persistent symptoms and health-related quality of life after hospitalization for COVID-19. Journal of Infection. 2020; 81: e4-e6.

[10] Ellul MA, Benjamin L, Singh B, Lant S, Michael BD, Easton A, et al. Neurological associations of COVID-19. Lancet Neurology. 2020; 19: 767-783.

[11] Carod Artal FJ. Complicaciones neurológicas por coronavirus y COVID19. Revista De Neurología. 2020; 70: 311-322.

[12] Bridwell R, Long B, Gottlieb M. Neurologic complications of COVID19. American Journal of Emergency Medicine. 2020; 38: 1549.e31549.e7.

[13] Kochi AN, Tagliari AP, Forleo GB, Fassini GM, Tondo C. Cardiac and arrhythmic complications in patients with COVID-19. Journal of Cardiovascular Electrophysiology. 2020; 31: 1003-1008.

[14] Inciardi RM, Lupi L, Zaccone G, Italia L, Raffo M, Tomasoni D, et al. Cardiac Involvement in a Patient with Coronavirus Disease 2019 (COVID-19). Journal of the American Medical Association Cardiology. 2020; 5: 819-824.

[15] Haimei M. Pathogenesis and Treatment Strategies of COVID-19-Related Hypercoagulant and Thrombotic Complications. Clinical and Applied Thrombosis/Hemostasis. 2020; 26: 107602962094449.

[16] Huang C, Huang L, Wang Y, Li X, Ren L, Gu X, et al. 6-month consequences of COVID-19 in patients discharged from hospital: a cohort study. Lancet. 2021; 397: 220-232.

[17] Richardson S, Hirsch JS, Narasimhan M, Crawford JM, McGinn T, Davidson KW, et al. Presenting Characteristics, Comorbidities, and Outcomes among 5700 Patients Hospitalized with COVID-19 in the New York City Area. Journal of the American Medical Association. 2020; 323: 2052-2059.

[18] Zhou F, Yu T, Du R, Fan G, Liu Y, Liu Z, et al. Clinical course and risk factors for mortality of adult inpatients with COVID-19 in Wuhan, China: a retrospective cohort study. Lancet. 2020; 395: 1054-1062.

[19] Somani SS, Richter F, Fuster V, De Freitas JK, Naik N, Sigel K, et al. Characterization of Patients who Return to Hospital Following Discharge from Hospitalization for COVID-19. Journal of General Internal Medicine. 2020; 35: 2838-2844.

[20] Kilaru AS, Lee K, Snider CK, Meisel ZF, Asch DA, Mitra N, et al. Return Hospital Admissions among 1419 COVID-19 Patients Discharged from Five U.S. Emergency Departments. Academic Emergency Medicine. 2020; 27: 1039-1042.

[21] Identificadas las principales manifestaciones en la piel de la COVID-19. British Journal of Dermatology. 2020; 183: e17.

[22] Wambier CG, Vaño-Galván S, McCoy J, Gomez-Zubiaur A, Herrera S, Hermosa-Gelbard A, et al. Androgenetic alopecia present in the majority of patients hospitalized with COVID-19: the "Gabrin sign". Journal of the American Academy of Dermatology. 2020; 83: 680-682.

How to cite this article: Álvaro Romero-Duarte, Mario Rivera-Izquierdo, Antonio Jesús Láinez-Ramos-Bossini, Pablo Redruello-Guerrero, Antonio Cárdenas-Cruz. Factors associated with readmission to the Emergency Department in a cohort of COVID-19 hospitalized patients. Signa Vitae. 2022;18(1):47-54. doi:10.22514/sv.2021.106. 